\title{
Modeling of kilometer-scale ionospheric irregularities at Mars
}

\author{
ChunHua Jiang*, Rong Tian, LeHui Wei, GuoBin Yang, and ZhengYu Zhao \\ School of Electronic Information, Wuhan University, Wuhan 430072, China
}

\section{Key Points:}

- Smaller-scale ionospheric irregularities were simulated in this model than in previous studies.

- Electric field shear between the topside and bottomside ionosphere occurs in the simulation when the plasma density is perturbed.

- Our simulations yield kilometer-scale irregularities that are comparable to those observed by MAVEN (Mars Atmosphere and Volatile EvolutioN).

Citation: Jiang, C. H., Tian, R., Wei, L. H., Yang, G. B., and Zhao, Z. Y. (2022). Modeling of kilometer-scale ionospheric irregularities at Mars. Earth Planet. Phys., 6(2), 213-217. http://doi.org/10.26464/epp2022011

\begin{abstract}
Recently, kilometer-scale Martian ionospheric irregularities have been measured by the Mars Atmosphere and Volatile EvolutioN (MAVEN) mission (Fowler et al., 2020). In this study, we carried out a simulation of these irregularities, assuming a uniform Martian zonal neutral wind and a cosinusoidal perturbation of the plasma density as the seeding source. Results show that a vertical electric field shear could be induced by such a plasma density perturbation. We find that the vertical electric field shear causes a velocity shear of the plasma between the topside and bottomside ionosphere, which in turn is able to produce kilometer-scale ionospheric irregularities - irregularities of a smaller scale than were seen in previous simulations (Jiang CH et al., 2021). These kilometer-scale variations with altitude, in plasma density and magnetic field profiles, are comparable to the MAVEN observations.
\end{abstract}

Keywords: Martian ionosphere; ionospheric irregularities; numerical simulation

\section{Introduction}

The Mars Atmosphere and Volatile EvolutionN (MAVEN) spacecraft were launched in November 2013. One of the orbiter's objectives is to study the structure of the Martian thermosphere (upper atmosphere) and ionosphere (Jakosky et al., 2015), in particular the poorly understood nightside ionosphere and small scale structure of the planet's ionosphere. It is well known that ionospheric irregularities in the $\mathrm{E}$ and $\mathrm{F}$ regions of the terrestrial ionosphere can be observed frequently by remote sensing, in situ measurement, and airglow observation techniques, etc. The scale of Earth's ionospheric irregularities ranges from sub-meter to hundreds of kilometers (Fejer and Kelley, 1980; Kelley, 2009; Peng YX et al., 2021). The large-scale structure (tens to hundreds of kilometers) of the Martian ionosphere is mainly magnetically controlled by crustal magnetic field sources (Duru et al., 2006; Andrews et al., 2015). However, small-scale ionospheric irregularities of Mars have not been studied sufficiently to reveal their characteristics and physical mechanisms.

Recently, Fowler et al. (2017) reported the first MAVEN observations of small-scale ionospheric irregularities (at scales of 15-20 km or less) in the Martian lower ionosphere. Harada et al. (2018) also report observations by the Mars Advanced Radar for Subsurface and lonospheric Sounding instrument on the Mars Express of

Correspondence to: C. H. Jiang, chuajiang@whu.edu.cn

Received 03 AUG 2021; Accepted 21 OCT 2021.

Accepted article online 25 JAN 2022.

(C)2022 by Earth and Planetary Physics. ionospheric irregularities/diffuse echoes. They suggest that gravity waves at Mars might play a significant role in the formation of these ionospheric irregularities. Fowler et al. (2019) have further investigated the characteristics of small-scale ionospheric irregularities. They found that the horizontal magnetic field, the SZA (Solar Zenith Angle) around 75-120 degrees, and outside of the strong crustal magnetic field, might be favorable conditions for small scale ionospheric irregularities. Kilometer-scale ionospheric irregularities were further reported by Fowler et al. (2020). Through a linear model, Keskinen (2018) suggested that the Martian ionosphere in the dynamo region might be unstable due to electromagnetic gradient drift instability. Furthermore, Jiang $\mathrm{CH}$ et al. (2021) carried out a nonlinear simulation of ionospheric irregularities, seeded by perturbation zonal winds, in a neutral wind frame (the background zonal wind is set to be zero); their numerical simulations yielded small-scale irregularities (less than the $25 \mathrm{~km}$ wavelength of the perturbation wind) but failed to produce smaller-scale (kilometer-scale) variations in electron density and magnetic field profiles comparable to those observed by MAVEN.

The purpose of this study is to reproduce the kilometer-scale variations in electron density and magnetic field profiles. Our nonlinear model assumes a uniform zonal neutral wind (not perturbative zonal winds) and the seeding source of ionospheric irregularities is chosen to be a cosinusoidal perturbation of the electron density, which could be induced by gravity waves in the Martian thermosphere (Yiğit et al., 2015; Terada et al., 2017). Details of our model and associated assumptions are described in Section 2. 
Results from the model and analysis are presented in Section 3. Section 4 compares results from our model with results from other simulations and observations. Section 5 summarizes our conclusions.

\section{Model Description}

Similar to the model of terrestrial ionospheric irregularities (Jiang $\mathrm{CH}$ et al., 2020), Jiang $\mathrm{CH}$ et al. (2021) developed a two-dimension nonlinear model to simulate ionospheric irregularities at Mars. In their model, the ion species and neutral species in the Martian dynamo region are, respectively, $\mathrm{O}_{2}^{+}$and $\mathrm{CO}_{2}$. Their model solves continuity and momentum equations for $\mathrm{O}_{2}^{+}$and electrons assuming a divergence-free current and a horizontal background magnetic field. The diamagnetic effect-the sum of magnetic and plasma pressure tends to be constant (Lühr et al., 2003)—was used to calculate the variation of magnetic field. The background electric field was set to be zero, and only the perturbation electric field was considered. In this model, the $X, Y, Z$ directions represent the zonal, meridional, and vertical directions, respectively. A twodimensional Cartesian $(X, Z)$ grid was applied in this study. The horizontal magnetic field is parallel to the $Y$ direction. The grid consists of 101 points in the zonal direction $(X)$ and 201 points in the vertical direction $(Z)$, with grid spacing of $500 \mathrm{~m}$ in the $X$ and 200 $\mathrm{m}$ in the $Z$ directions. Periodic boundary conditions were imposed in the $X$ direction. Transmittive boundary conditions were imposed in the $Z$ direction. The running time step is set to $0.01 \mathrm{~s}$ in the simulation. Details of the model and numerical schemes are described in Jiang CH et al. (2021).

Unlike the study by Jiang $\mathrm{CH}$ et al. , the initial seeding source in this study is a cosinusoidal perturbation of the electron density. England et al. (2017) reported atmospheric gravity waves in the Martian thermosphere with scale sizes in the tens to hundreds of kilometers. Therefore, we set the wavelength of the perturbation in our model at $25 \mathrm{~km}$. MAVEN observations of the Martian ionosphere (Fowler, et al., 2017) have shown that large relative fluctu- ations (10-50\%) in plasma density $\left(\delta n_{\mathrm{e}} / n_{\mathrm{e}}\right)$ and magnetic field $(\delta B / B)$ can occur. Therefore, we find it reasonable to set the amplitude of perturbation density to $5 \%$ in this simulation when gravity waves occur in the Martian thermosphere (Fritts et al., 2006; Yiğit et al., 2015; Terada et al., 2017; Keskinen, 2018). The background zonal wind is set to be $100 \mathrm{~m} / \mathrm{s}$ and is kept uniformly horizontal. The initial magnetic field strength is set to be $10 \mathrm{nT}$. Because ionospheric irregularities are observed to occur primarily at solar zenith angles of $\sim 90-130$ degrees, in this model we set the peak $\mathrm{O}_{2}^{+}$density to be $3 \times 10^{3} \mathrm{~cm}^{-3}$ and the corresponding scale height to be $15 \mathrm{~km}$. The background plasma density and magnetic field in this model are comparable to MAVEN observations (Fowler et al., 2017, 2019).

\section{Results}

Figure 1 shows the profiles of the plasma density (left panel), electric potential (middle panel), and magnetic field (right panel) at the beginning $(t=1 \mathrm{~s})$ of this simulation. The initial plasma density is subject to a cosinusoidal perturbation, which is the seeding source of ionospheric irregularities. Due to the assumption in this model that the sum of magnetic and plasma pressures tends to be constant when the magnetic field is horizontal, the magnetic field fluctuations therefore occur out of phase with the plasma density. It can be seen from Figure $1 \mathrm{~b}$ that the electric potential fluctuations occur at the beginning stage. The electric potential distribution in the uniform wind is different from that in the previous simulation (Jiang CH et al., 2021). In the Martian ionosphere, the ion-neutral collision is greater than the corresponding gyrofrequency but inverse for electrons. Neutral winds could lead to the separation of the ions and electrons in the dynamo region. Therefore, plasma density fluctuations could plausibly induce a polarization electric field in the Martian ionosphere. We have found that both vertical and zonal electric fields could occur in the plasma density fluctuations. In particular, stronger electric potential perturbations emerge at the bottomside ionosphere due to greater ion-neutral collisions. Obviously, the vertical electric field shear
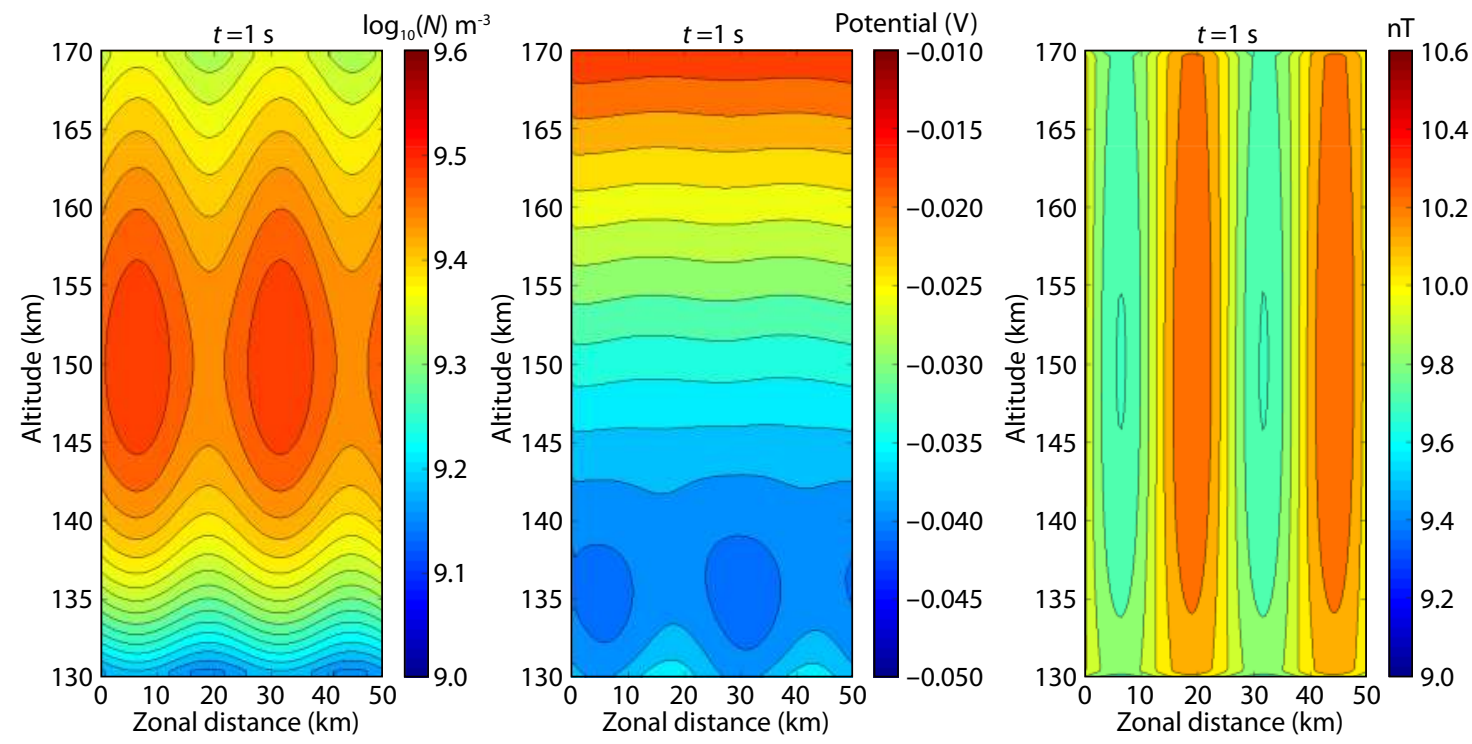

Figure 1. Plasma density (left), electric potential (middle) and magnetic field (right) distribution simulated at the beginning stage $(t=1 \mathrm{~s})$. 
could thus be produced between the bottomside and topside ionosphere. Because the electrons are mostly magnetized in the Martian ionosphere but not the ions, to further demonstrate the distribution of the electric field we have assumed that the positive ions can move away with neutral winds but not the electrons. Accordingly, in the higher plasma density region, more ions will be blown away leaving an excess of electrons. As a result, electrons in the lower plasma density region will be fewer, compared with the higher density region. Furthermore, the potential in the higher density region is lower than in the lower density region. This assumption is consistent with the distribution of the electric potential shown in Figure 1b. However, we should keep in mind that both the plasma density and ion-neutral collisions will play significant roles in determining where the lower potential occurs. Thus, the altitudinal variations of plasma density and ion-neutral collisions could lead to altitudinal variations of electric potential. It also should be noted that the overall electric potential is negative in Figure 1. The negative potential is associated with the effect of the neutral wind. However, the potential difference (the electric field) plays a significant role in the movement of the $\mathrm{O}_{2}^{+}$ions and electrons in the plasma.

Figure 2 shows the nonlinear evolution of the plasma density simulated at $t=0,100,300,500,700$, and $1000 \mathrm{~s}$. It can be seen from Figure 2 that at $t=100 \mathrm{~s}$ the lower plasma density moves upward into the higher plasma density region. Due to gradient drift instability in the Martian dynamo region, the zonal electric field induced by plasma density perturbations and neutral winds leads the lower plasma density into higher density regions. After $t=300 \mathrm{~s}$, it is seen clearly that velocity shear of the plasma between the topside and bottomside ionosphere is occurring. The topside plasma moves eastward but the bottomside plasma is moving westward. It also can be seen from Figure $1 \mathrm{~b}$ that the vertical electric field shear occurs at the altitude of $\sim 135 \mathrm{~km}$. As a result, there are plenty of smaller-scale irregularities in the bottomside ionosphere (see $t=500,700$, and $1000 \mathrm{~s}$ in Figure 2). Figure 3 demon- strates the evolution of the associated magnetic field in the present simulation. Similar to Figure 2, plenty of smaller-scale magnetic irregularities can be observed in the bottomside ionosphere (at $t=500,700,1000 \mathrm{~s}$ ). According to the results of the simulation (see Figures 2 and 3), the scale of the irregularity is first associated with the wavelength of the perturbation in the plasma. Then, the most important factor is the velocity shear of the plasma, which leads to Kelvin-Helmholtz instability. At the beginning of the simulation ( $t=100 \mathrm{~s}$ in Figure 2 ), the scale of the irregularity corresponds to the wavelength of the perturbation. When the velocity shears of the plasma become stronger in the bottomside of the ionosphere, the scale of the irregularity becomes smaller (see $t=300,500,700,1000 \mathrm{~s}$ in Figure 2). In other words, the perturbative electric field is induced by the initial plasma density perturbation; then a Kelvin-Helmholtz instability (caused by the velocity shear of the plasma) leads to smaller-scale irregularity.

Figure 4 shows the variations of electron density and magnetic fields with altitude (Figures $4 \mathrm{a}$ and $4 \mathrm{~b}$ ) at the zonal distance of $20 \mathrm{~km}$. The red and blue lines, respectively, represent the running time of 700 and $1000 \mathrm{~s}$. It can be seen from Figure 4 that there are many smaller-scale 'oscillations' occurring along the altitude. As the time progresses, smaller-scale perturbations occur at the topside ionosphere (see also Figure 2, at $t=700,1000 \mathrm{~s}$ ).

\section{Discussion}

MAVEN observations of small-scale ionospheric irregularities were studied by Fowler et al. (2017, 2019), who reported many smallerscale (kilometer-scale) 'oscillations' along the track of the spacecraft (in both the vertical and horizontal directions). Keskinen (2018) was first to analyze these ionospheric irregularities linearly (both the plasma density and magnetic fluctuations), proposing that the MAVEN observations might be explained by plasma electromagnetic gradient drift instability. Subsequently, Jiang $\mathrm{CH}$ et al. (2021) carried out a nonlinear simulation of the Martian ionospheric irregularties in a neutral wind frame (setting the back-
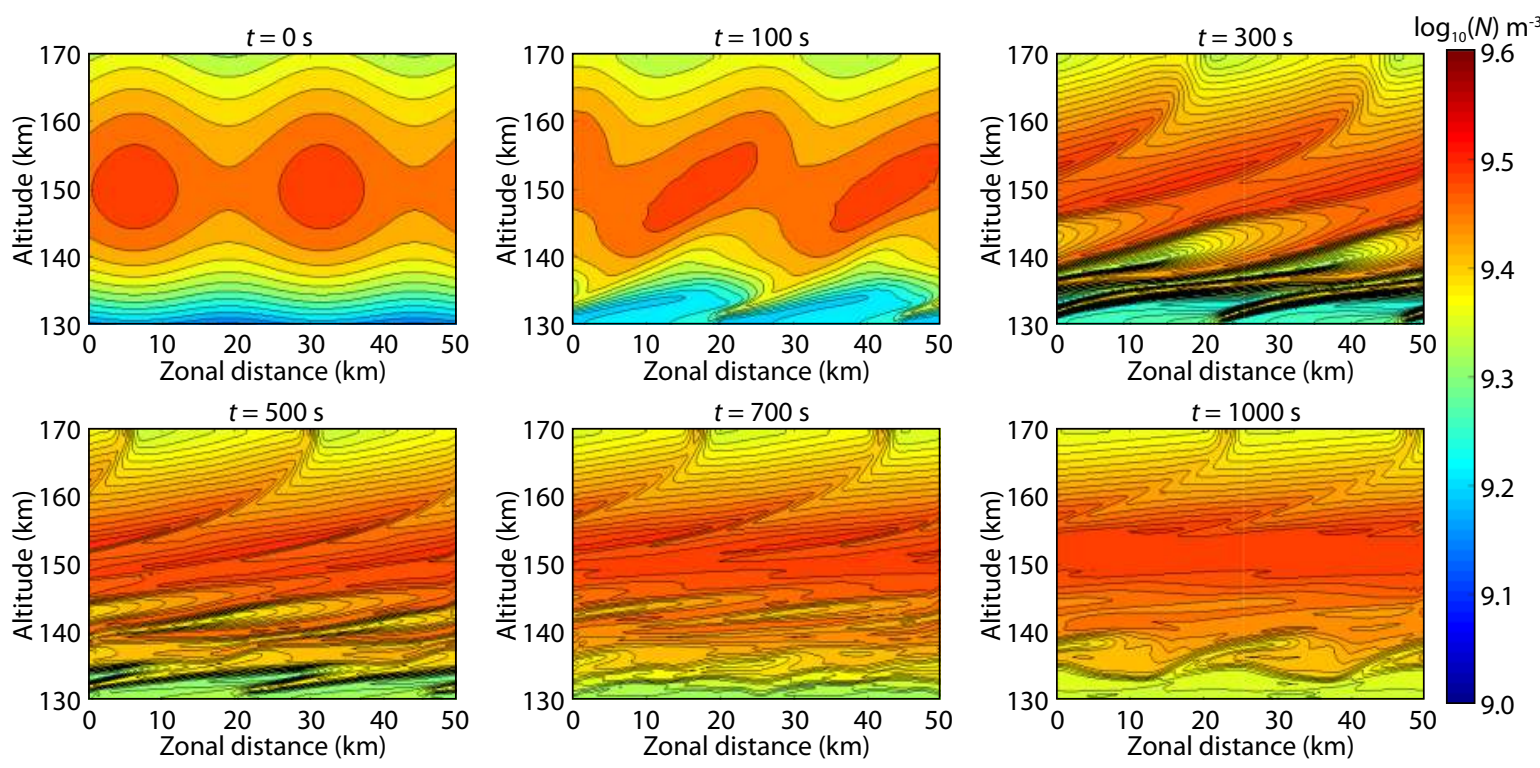

Figure 2. Plasma density distribution simulated at $t=0,100,300,500,700$, and $1000 \mathrm{~s}$. 

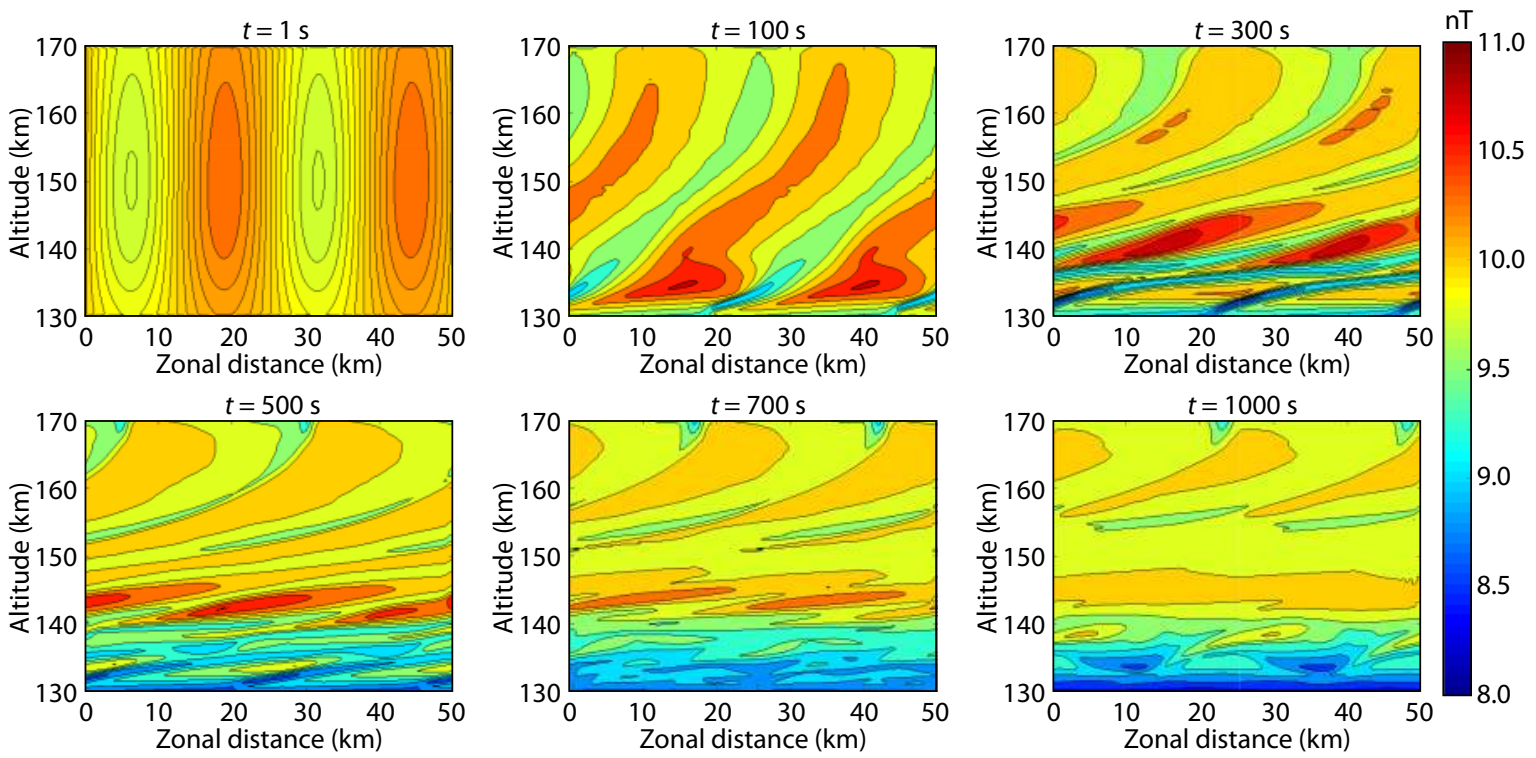

Figure 3. Similiar to Figure 2 but for magnetic field.
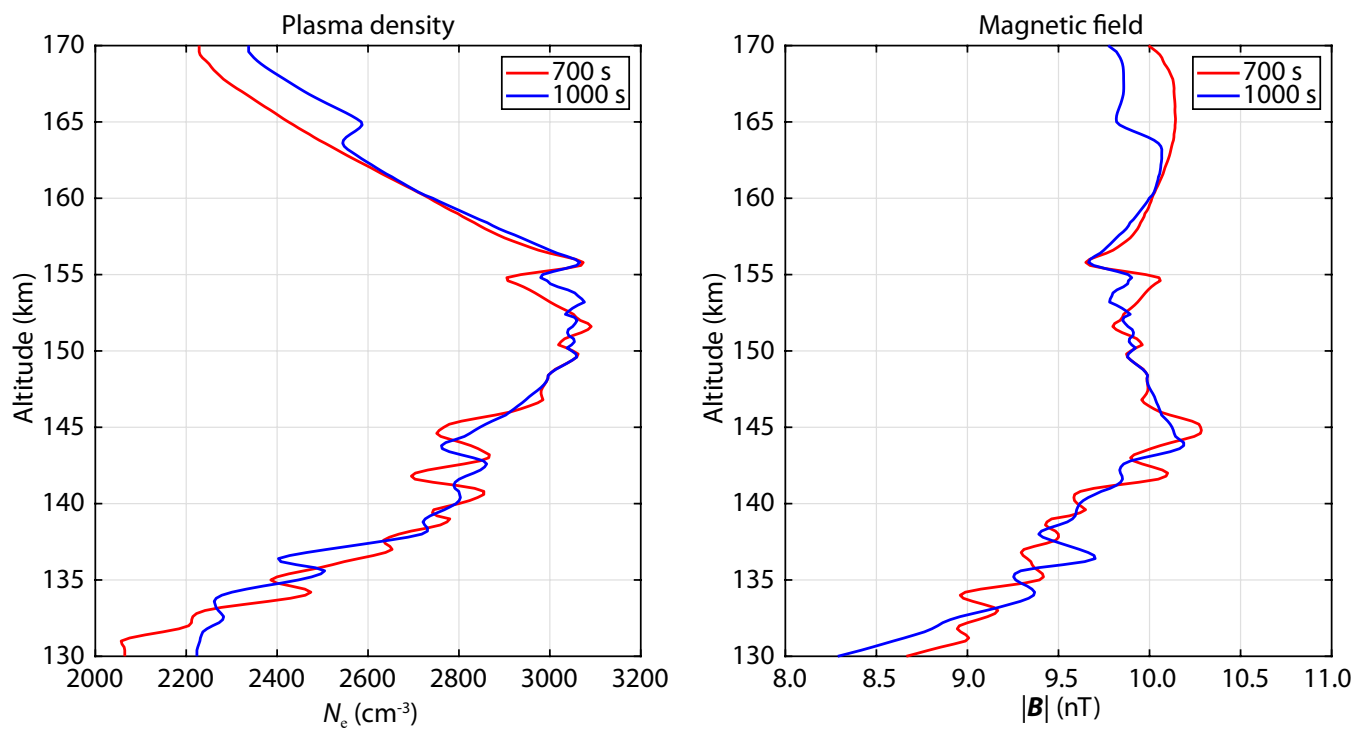

Figure 4. Vertical profiles of plasma density and magnetic field in this simulation at the zonal distance of $20 \mathrm{~km}$ at $t=700 \mathrm{~s}$ (red), $1000 \mathrm{~s}$ (blue).

ground wind at zero). However, the neutral wind frame simulation did not reproduce the observed altitudinal (vertical direction) kilometer-scale ionospheric irregularities. Fowler et al. (2020) reported that the kilometer-scale 'oscillations' structure in the irregularity profiles (vertical direction) can be observed both above and below the ionospheric peak, and pointed out further that two kinds of kilometer-scale irregularities appear in the Martian ionosphere. The density and magnetic field fluctuations vary in and out of phase. In the present simulation, diamagnetic effects are considered. Thus, only out of phase irregularities could be simulated. Figure 4 reveals many kilometer-scale irregularities (out of phase irregularities) reproduced in the profiles of the plasma density and magnetic field, especially below $\sim 155 \mathrm{~km}$. Moreover, the scale of irregularities becomes much smaller around 150$155 \mathrm{~km}$. In the present simulation, the kilometer-scale irregularit- ies in the profiles of the plasma density and magnetic field are comparable to MAVEN observations.

Keskinen (2018) pointed out that the dynamo region of the Martian ionosphere is unstable due to gradient drift instability in the neutral wind. In fact, the growth rates of irregularities in the Martian ionosphere depend on many factors including the neutral wind, the amplitude wavelength of the perturbation in the plasma, the background plasma density, the collision frequency, the magnetic field strength, etc. By calculating the growth rates from the perturbation in the ionospheric plasma density, Keskinen (2018) found that the growth time for irregularities ranged from about 50 to $1000 \mathrm{~s}$ for the wavelengths of several kilometers to $\sim 20 \mathrm{~km}$. Figure 4 shows that in our simulation many smallerscale (kilometer-scale) 'oscillations' occur along the altitude at running times of 700 and $1000 \mathrm{~s}$. The growing time of the irregu- 
larity in this simulation is consistent with the linear theory proposed by Keskinen (2018). In addition, Keskinen (2018) suggests that the ionosphere is unstable below the ionospheric peak, also pointed out by Fowler et al. (2019). In the present simulation, the ionospheric peak is set at $150 \mathrm{~km}$. However, we found that ionospheric irregularities could reproduced above the ionospheric peak $(150 \mathrm{~km})$ at $t=1000 \mathrm{~s}$. In the present simulation, the velocity shear of the plasma (the background neutral wind is set to be uniform) could lead to the Kelvin-Helmholtz instability. Furthermore, the Kelvin-Helmholtz instability produces kilometer-scale plasma density and magnetic irregularities below and above the ionospheric peak. Although the nonlinear model of Martian ionospheric irregularities presented here is simplified by the background parameters of the ionosphere and upper atmosphere, this simplified model can help us understand the nonlinear evolution of ionospheric irregularities at Mars. It is encouraging that these modeling results are comparable to MAVEN observations of kilometer-scale irregularities.

\section{Conclusion}

In this study, we carried out a numerical simulation of kilometerscale irregularities in the Martian ionosphere. A uniform zonal wind was adopted in this model. The seeding source is a cosinusoidal perturbation of plasma density. Modeling results show that the vertical electric field shear can be produced by perturbation of the plasma. The electric field shear in the ionosphere leads to a velocity shear of the plasma between the topside and bottomside ionosphere. Kilometer-scale altitudinal (vertical direction) irregularities are reproduced in the present simulation. Results show that the simulated altitudinal variations of kilometer-scale irregularities in plasma density and magnetic field are comparable to those seen in MAVEN observations.

\section{Acknowledgments}

This work was supported by the National Natural Science Foundation of China (NSFC No.42074184 and No.41727804). The numerical code used for this study is not prepared for publicly accessible servers. The data used in this study are available from Zenodo: https://zenodo.org/record/5052664 (DOI: 10.5281/zenodo.5052 664 , the section: Numerical Simulation of Small-Scale lonospheric Irregularities at Mars).

\section{References}

Andrews, D. J., Andersson, L., Delory, G. T., Ergun, R. E., Eriksson, A. I., Fowler, C. M., McEnulty, T., Morooka, M. W., Weber, T., and Jakosky, B. M. (2015). lonospheric plasma density variations observed at Mars by MAVEN/LPW. Geophys. Res. Lett., 42(21), 8862-8869. https://doi.org/10.1002/ 2015GL065241

Duru, F., Gurnett, D. A., Averkamp, T. F., Kirchner, D. L., Huff, R. L., Persoon, A. M., Plaut, J. J., and Picardi, G. (2006). Magnetically controlled structures in the ionosphere of Mars. J. Geophys. Res., 111(A12), A12204.

https://doi.org/10.1029/2006JA011975
England, S. L., Liu, G., Yiğit, E., Mahaffy, P. R., Elrod, M., Benna, M., Nakagawa, H., Terada, N., and Jakosky, B. (2017). MAVEN NGIMS observations of atmospheric gravity waves in the Martian thermosphere. J. Geophys. Res., 122(2), 2310-2335. https://doi.org/10.1002/2016JA023475

Fejer, B. G., and Kelley, M. (1980). lonospheric irregularities. Rev. Geophys., 18(2), 401-454. https://doi.org/10.1029/RG018i002p00401

Fowler, C. M., Andersson, L., Shaver, S. R., Thayer, J. P., Huba, J. D., Lillis, R., Usanova, M. E., Espley, J., Ergun, R. E., ... Jakosky, B. M. (2017). MAVEN observations of ionospheric irregularities at Mars. Geophys. Res. Lett., 44(21), 10845-10854. https://doi.org/10.1002/2017GL075189

Fowler, C. M., Bonnell, J. W., Huba, J. D., Andersson, L., Benna, M., and Ergun, R. E. (2019). The statistical characteristics of small-scale ionospheric irregularities observed in the Martian ionosphere. J. Geophys. Res., 124(7), 5874-5893. https://doi.org/10.1029/2019JA026677

Fowler, C. M., Bonnell, J. W., Xu, S., Benna, M., Elrod, M., McFadden, J., Mitchell, D. L., Espley, J., Andersson, L., ... Jakosky, B. (2020). First detection of kilometer-scale density irregularities in the Martian ionosphere. Geophys. Res. Lett., 47(22), e2020GL090906. https://doi.org/10.1029/2020GL090906

Fritts, D. C., Wang, L., and Tolson, R. H. (2006). Mean and gravity wave structures and variability in the Mars upper atmosphere inferred from Mars Global Surveyor and Mars Odyssey aerobraking densities. J. Geophys. Res., 111(A12), A12304. https://doi.org/10.1029/2006JA011897

Harada, Y., Gurnett, D. A., Kopf, A. J., Halekas, J. S., and Ruhunusiri, S. (2018). lonospheric irregularities at Mars probed by MARSIS topside sounding. J. Geophy. Res., 123(1), 1018-1030. https://doi.org/10.1002/2017JA024913

Jakosky, B. M., Lin, R. P., Grebowsky, J. M., Luhmann, J. G., Mitchell, D. F., Beutelschies, G., Priser, T., Acuna, M., Andersson, L., ... Zurek, R. (2015). The Mars Atmosphere and Volatile Evolution (MAVEN) mission. Space Sci. Rev., 195(1), 3-48. https://doi.org/10.1007/s11214-015-0139-x

Jiang, C. H., Wei, L. H., Yang, G. B., Zhou, C., and Zhao, Z. Y. (2020). Numerical simulation of the propagation of electromagnetic waves in ionospheric irregularities. Earth Planet. Phys., 4(6), 565-570. https://doi.org/10.26464/epp2020059

Jiang, C. H., Yokoyama, T., Wei, L. H., Yang, G. B., and Zhao, Z. Y. (2021) Nonlinear simulation of ionospheric irregularities at mars. Astrophys. J., 909(1), 47. https://doi.org/10.3847/1538-4357/abdc1d

Kelley, M. C. (2009). The Earth's lonosphere: Plasma Physics and Electrodynamics (2nd ed). San Diego: Academic Press.

Keskinen, M. J. (2018). New model for ionospheric irregularities at Mars. Geophys. Res. Lett., 45(5), 2177-2183. https://doi.org/10.1002/ 2017GL076507

Lühr, H., Rother, M., Maus, S., Mai, W., and Cooke, D. (2003). The diamagnetic effect of the equatorial Appleton anomaly: its characteristics and impact on geomagnetic field modeling. Geophys. Res. Lett., 30(17), 1906. https://doi.org/10.1029/2003GL017407

Peng, Y. X., Scales, W. A., Hartinger, M. D., Xu, Z. H., and Coyle, S. (2021). Characterization of multi-scale ionospheric irregularities using groundbased and space-based GNSS observations. Satell. Navig., 2(1), 14. https://doi.org/10.1186/s43020-021-00047-x

Terada, N., Leblanc, F., Nakagawa, H., Medvedev, A. S., Yiğit, E., Kuroda, T., Hara, T., England, S. L., Fujiwara, H., ... Jakosky, B. M. (2017). Global distribution and parameter dependences of gravity wave activity in the Martian upper thermosphere derived from MAVEN/NGIMS observations. J. Geophys. Res., 122(2), 2374-2397. https://doi.org/10.1002/2016JA023476

Yiğit, E., England, S. L., Liu, G. P., Medvedev, A. S., Mahaffy, P. R., Kuroda, T., and Jakosky, B. M. (2015). High-altitude gravity waves in the Martian thermosphere observed by MAVEN/NGIMS and modeled by a gravity wave scheme. Geophys. Res. Lett., 42(21), 8993-9000. https://doi.org/10.1002/ 2015GL065307 